\title{
TORQUE CONTROL OF AC MOTOR WITH FOPID CONTROLLER BASED ON FUZZY NEURAL ALGORITHM
}

\author{
Gholamreza Farahani \\ Department of Electrical Engineering and Information Technology, Iranian Research \\ Organization for Science and Technology (IROST), Tehran, Iran
}

\begin{abstract}
Nowadays in the complicated systems, design of proper and implementable controller has a most importance. With respect to ability of fractional order systems in complicated systems identification as a first order fractional system with time delay, usage of fractional order PID has a proper result. From one side flexibility of fractional calculus than integer order has been topics of interest to the researchers. From another side, PMSM motors which are one the AC motor types, has been allocated largely accounted position in industry and used in variety applications. Therefore in this paper torque direct control of PMSM motors with FOPID based on model is proposed. Also fuzzy neural controllers are widely considered. Reason of this is success of fuzzy neural controller in control and identification of uncertain and complicated systems. The proposed method in this paper is combination of FOPID controller with fuzzy neural supervision system which with coefficients setting of this controller, control operation of PMSM will improve. Results of proposed method show the ability of proposed technique in reference signal tracking, elimination of disturbances effects and functional robustness in presence of noise and uncertainty. The results show the error averagely in three condition, nominal form, step disturbance and noise and uncertainly will decrease $11.66 \%$ in proposed method (FNFOPID) with Integral Square Error criterion and $7.69 \%$ with Integral Absolute Error criterion in comparison to FOPID.
\end{abstract}

\section{KEYWORDS}

Fractional Calculus, Fractional Order PID Controller, Fuzzy Neural Systems, Torque Direct Control, Permanent Magnet Synchronous Motor.

\section{INTRODUCTION}

One of the essential requirements of industrial societies is usage of electricity to produce required mechanical energy of small and large industries. Electrical machines are the most important converters to convert electrical energy to mechanical and vice versa. Few people can be found which is not dealt with different electrical machines. Every morning many people unconsciously start up their cars DC motor. Most families use a cooling device which its cold achieves from compressing gas inside of it with one phase or three phase induction motor. Usage of elevator can be seen in abundance among people. Nowadays modern agriculture without electric motors will become commonplace industry. In high-tech industry such as military, medicine and aerospace, precision electric motors are used in abundance which most of these motors have advanced control system. Generally any where rotating or linear motion of load is required, use of electric motor with an advanced control system is inevitable.

Permanent Magnet Synchronous Motors (PMSM) due to the high power density, high ratio of torque to inertia, fast acceleration, easier maintenance operations, better power factor and efficiency have preferred than direct current and induction motors in many industrial applications at low and medium power range. 
In fact PMSM is a conventional synchronous motor which instead of excitation rotor winding, brushes and slider rings, the permanent magnet is used. Permanent magnet synchronous motor stator is like as induction motors and its required (Electromotive force) EMF is sinusoidal. In PMSM motors, stator current should be sinusoidal till uniform torque achieve in motors. After simplifications, dynamic model of PM motor in rotary Quasi-Dipole (QD) coordinate is as below [1].

$$
\begin{aligned}
& v_{q s}^{r}=r_{s} i_{q s}^{r}+\omega_{r} \lambda_{d s}^{r}+\frac{d}{d t} \lambda_{q s}^{r} \\
& v_{d s}^{r}=r_{s} i_{d s}^{r}-\omega_{r} \lambda_{q s}^{r}+\frac{d}{d t} \lambda_{d s}^{r} \\
& \lambda_{q s}^{r}=L_{q s} i_{q s}^{r} \\
& \lambda_{d s}^{r}=L_{d s} i_{d s}^{r}+\lambda_{m}^{r} \\
& L_{q s}=L_{l s}+L_{m q} \\
& L_{d s}=L_{l s}+L_{m d} \\
& T_{e}=\frac{3}{2} \frac{P}{2}\left[\lambda_{m}^{r} i_{q s}^{r}+\left(L_{d s}-L_{q s}\right) i_{q s} i_{d s}\right]
\end{aligned}
$$

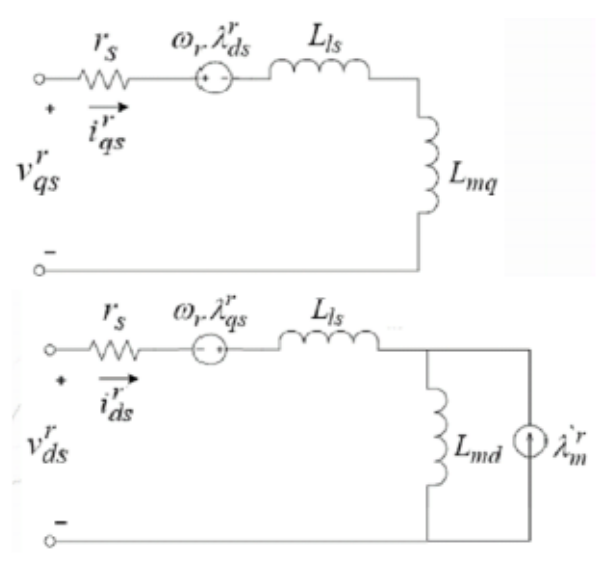

where $L_{d s}$ is a direct-axis inductance, $L_{q s}$ is a quadrature-axis inductance, $\omega_{r}$ is electrical rotor angular speed, $v_{q s}^{r} \mathrm{q}$-axis control input, $v_{d s}^{r} \mathrm{~d}$-axis control input, $i_{q s}^{r} \mathrm{q}$-axis current, $i_{d s}^{r} \mathrm{~d}$-axis current, $r_{s}$ stator resistance, $L_{m q}$ q-axis magnetic inductance, $L_{m d}$ d-axis magnetic inductance, $L_{l s}$ stator inductance, $\lambda_{q s}^{r}$ q-axis magnetic flux, $\lambda_{d s}^{r} \mathrm{~d}$-axis magnetic flux, $\lambda_{m}^{r}$ magnetic flux, $P$ number of poles and $T_{e}$ is output torque. As is evident from the equations (1), the resulting torque, will include two parts originate from PM and reluctance torque. In synchronous motors with surface permanent magnet, $L_{d}$ and $L_{q}$ are equal, therefore reluctance torque term will not exist. In internal magnet type $L_{d}<L_{q}$, therefore positive current $i_{d s}$ will reduce the torque which should be omitted with design of proper controller

\section{PMSM MOTORS CONTROL METHODS}

There are different methods to control PMSM motors. In this section some of these techniques will be investigated.

\subsection{Control With Stable V/F Scalar Method}

This method [2] makes keeping the flux magnitude in the nominal value of permanent magnet synchronous motor. This control technique is a simple, cheap and industrial method to control AC motors including PMSM. It should be noted because of synchronous motors capability, there is no need to design closed loop speed control in V/F method.

\subsection{VECTOR CONTROL}

In this control technique [3-5] it is tried that the rotor flux always be on the d-axis. With regard to the permanent magnet on the rotor, rotor flux always exits and is aligned with the d-axis. Therefore in the absence of the need to reduce flux produced with permanent magnet (it is 
necessary for higher speeds than nominal speed), flux component produced with $i_{d s}$ should be zero till torque is not reduced.

\subsection{Direct Torque CONTROL (DTC) METHOD}

This technique with use of designed controllers, based on flux and torque error, directly commanded to inverter. In this method required torque and flux of controllers will calculate in the estimator block. With respect to results, response time of DTC in tracking is more than vector control for control of permanent magnet motor and also DTC is better than vector control method in suppression the effect of disturbances $[6,7]$. To estimate flux and torque in this control mechanism, different method is used such as neural network, fuzzy, Luenberger observer, Model Reference Adaptive System (MRAS) estimator, Kalman filter, Sliding Mode Control (SMC) or combination of these techniques.

\section{Fractional ORder PID (FOPID) CONTROLLER}

In this section different types of FOPID controllers will be presented.

\subsection{INTRODUCING PID CONTROLLER FAMILY}

Five types of controller can be considered in this controller family which are (ProportionalIntegral) PI, (Proportional-Derivative) PD, (Proportional-Integral-Derivative) PID, Lead and Lag. Each controller has special features. If structure of these controllers extended as fractional form, it will be as equation (2).

$$
\begin{aligned}
& P I: C_{P I}(s)=K_{p}+\frac{K_{i}}{s^{\lambda}} \\
& P D: C_{P D}=K_{p}+K_{d} s^{\mu} \\
& P I D: C_{P I D}=K_{p}+\frac{K_{i}}{s^{\lambda}}+K_{d} s^{u}
\end{aligned}
$$

where $K_{p}$ is Proportional gain, $K_{i}$ Integral gain, $K_{d}$ Derivative gain, $\lambda$ order of the integrator and $\mu$ order of the differentiator. Figure 1 shows the concept of extension from integer order space to fractional order space.


Figure 1. Extension from integer order to fractional order space

As shown in figure 1, integer order controller in fact is a special case of fractional order controllers. Therefore fractional order is taken into consideration which it has more flexibility and ability than integer order and may be in the design of controller with use of optimization methods, the points between zero and one is the answer of problem which it shows the importance of this subject. At continuation, introduction and definition of controllers design methods will describe. 


\subsection{Fractional Order Controllers Design}

\subsubsection{TAYLOR SERIES METHOD}

In [8] second order standard model for plant is considered and mathematically proved that it is not possible to get the closed loop response of system with integer order PID as a standard second order but with Taylor expansion method with consideration controller as fractional, the closed loop response will be possible to an acceptable level. PID controller's equations with the integer and fractional forms are as equation (3).

$$
C_{P I D_{\text {latager }}}=K_{c}\left(1+\frac{K_{i}}{s}+K_{d} s\right) \quad C_{P D_{\text {Praxiond }}}=K_{c}\left(1+\frac{K_{i}}{s^{\lambda}}+K_{d} s^{\mu}\right)
$$

Assume that desired closed loop is as below.

$$
G_{d}=\frac{\omega_{n}^{2}}{s^{2}+2 \xi \omega_{n}+\omega_{n}^{2}}
$$

where $\xi$ and $\omega_{n}$ are damping coefficient and natural frequency respectively. If desired plant is shown with $G_{p}$ symbol and controller with $G_{c}$ symbol, the closed loop response with unit negative feedbad will be:

$$
G_{\text {closedloop }}=\frac{G_{c} G_{p}}{1+G_{c} G_{p}}
$$

If the controller form is considered as below:

$$
G_{c}(s)=K_{c}\left(1+\frac{K_{i}}{s^{\lambda}}+K_{d} s^{\mu}\right)
$$

$\lambda$ and $\mu$ are the differentiator and integral parameters that in this case are not integer and their value is between 0 and 2 . If the fractional order controller form shows as an another form, then

$$
G_{c}(s)=K_{c}\left(1+\frac{K_{i}}{s^{\lambda}}+K_{d} s^{\mu}\right)=\frac{K_{c}}{s}\left(s+\frac{K_{i}}{s^{\lambda-1}}+K_{d} s^{\mu+1}\right)=\frac{K_{c}}{s} G_{c}(s)
$$

Therefore closed loop form will be as equation (8).

$$
\text { Closed loop }=\frac{G_{c} G_{p}}{1+G_{c} G_{p}}=\frac{\left(\frac{K_{c}}{s} G_{c}(s)\right) G_{p}}{1+\left(\frac{K_{c}}{s} G_{c}(s)\right) G_{p}}=\frac{K_{c} G_{c}(s) G_{p}}{s+K_{c} G_{c}(s) G_{p}}
$$

If general form of plant and controller insert into the equation (8), this form never be equal with the desired form $G_{d}$ and its reason is that approximation methods is used. Taylor series is one the most popular and simplest approximation methods. Because in the controller there is five unknown parameters, Taylor series will expand to five orders and ignore the rest sentences of series.

\subsubsection{DESIGn BASED ON STATE SPACE}

In this section we assume system transfer function is as equation (9). 
$G_{p}(s)=\frac{1}{a_{2} s^{\alpha}+a_{1} s^{\beta}+a_{0}}$

Now with the view of state space, FOPI and FOPD controllers will design. In the design of FOPD controllers we assume the controller form as equation (10).

$C(s)=K_{p}+K_{d} s^{\mu}$

Therefore in closed loop system the transfer function is:

$$
\begin{aligned}
& \text { closed loop }=\frac{G_{p} C}{1+G_{p} C}=\frac{K_{p}+K_{d} s^{\mu}}{a_{2} s^{\alpha}+a_{1} s^{\beta}+K_{d} s^{\mu}+\left(a_{0}+K_{p}\right)} \\
& =a_{2} y^{\alpha}(t)+a_{1} y^{\beta}(t)+K_{d} y^{\mu}(t)+\left(a_{0}+K_{p}\right) y(t)=K_{p} w(t)+K_{d} w^{\mu}(t)
\end{aligned}
$$

Now state equations of system are as below.

$$
\begin{gathered}
\left\{\begin{array}{c}
\dot{x}_{1}(t)=x_{2}(t) \\
\dot{x_{2}}(t)=-\frac{a_{0}+K_{p}}{a_{2}} x_{1}{ }^{2-\alpha}(t)-\frac{K_{d}}{a_{2}} x_{2}^{1+\mu-\alpha}(t) \\
-\frac{a_{1}}{a_{2}} x_{2}^{1+\beta-\alpha}(t)+\frac{1}{a_{2}} w^{2-\alpha}(t)
\end{array}\right. \\
z(t)=K_{p} x_{1}(t)+K_{d} x_{2}^{\mu-1}(t)
\end{gathered}
$$

With use of Laplace transform of equation (12) will be:

$$
\left\{\begin{array}{c}
s X(s)=A(s) X(s)+B(s) W(s) \\
Y(s)=C(s) X(s)
\end{array}\right.
$$

Where

$$
A(s)=\left[\begin{array}{cc}
0 & 1 \\
-\frac{a_{0}+K_{p}}{a_{2}} s^{2-\alpha} & -\frac{K_{d} s^{1+\mu-\alpha}+a_{1} s^{1+\beta-\alpha}}{a_{2}}
\end{array}\right]
$$

Therefore system characteristic equation which will obtain from $\operatorname{det}(s I-A)=0$ is as:

$$
a_{2} s^{\alpha}+a_{1} s^{\beta}+K_{d} s^{\mu}+\left(a_{0}+K_{p}\right)=0
$$

Now controller parameters can be determined in such a way closed loop poles placed on the desired poles

\subsubsection{OPTIMAL PID CONTROLLER}

In this controller due to five degrees of freedom, five constrains should be considered. For this reason this controller is one of the most widely controllers between FOPID controllers. In the 
design of this controller, it has been tried that tracking in addition to noise and distortion cancellation is considered which it shows the high flexibility of this controller [9].

\subsubsection{Fractional ORder LAg AND LEAD CONTROLler}

In the field of fractional order lag and lead controllers design, few studies have been carried out. General form of this controller will define as below [10].

$$
C(s)=K_{c}\left(\frac{s+\frac{1}{\lambda}}{s+\frac{1}{x \lambda}}\right)^{\alpha}=K_{c} x^{\alpha}\left(\frac{\lambda s+1}{\lambda x s+1}\right)^{\alpha} \quad(0<x<1)
$$

This controller in comparison to its integer counterpart has a new parameter $\alpha$, which is fractional order of controller. For $\alpha>0$ controller is lag and zero of controller is $\omega_{\text {zero }}=1 / \lambda$ and pole of controller is $\omega_{\text {pole }}=1 / x \lambda$. For $\alpha<0$ controller is lead.

\subsubsection{CONTRoller Design With USE OF Evolutionary AlgorithmS}

A group of researchers in the recent years has concentrated on the Genetic Algorithm (GA) and Particle Swarm Optimization (PSO) to set five fractional order parameters [11]. In this subsection, design method with GA will describe. In fact, GA method is base on minimization of cost function. Generally cost function defines as equation (16).

$$
J=\int_{0}^{\infty}\left(w_{1}|e(t)|+w_{2} u^{2}(t)\right) d t
$$

$w_{1}$ and $w_{2}$ coefficient is used to give an opportunity to the designer to decide which part of equation (16) is preferred. First part of equation (16) causes final error reduction and second part of it will reduce control effort. GA binary algorithm can be proper solution for minimization of cost function.

\section{Fuzzy And Neural Systems}

In this section, first fuzzy system will explain and then neural systems are described.

\subsection{TAKAGI-SUGENO FUZZY SYSTEMS}

Takagi-Sugeno fuzzy system in one of the methods similar to the multiple systems which in model output combination, fuzzy switching is used and this system will classify in the soft switching category. Also it is possible to use an expert knowledge in membership functions of Takagi-Sugeno fuzzy systems which in some cases such as nonlinear system identification will improve system performance. Takagi-Sugeno fuzzy models which proposed with Sugeno and classified in the third division of fuzzy models, in fact is a fuzzy dynamic model [12]. This model is based on set of fuzzy rules that with combining linear systems has a capability for description of complicated nonlinear systems. Other important feature of Takagi-Sugeno fuzzy models is the existence of a tool for systematic development to review stability and fuzzy controller design with use of theories and powerful and new control techniques. 
Takagi-Sugeno fuzzy models which use for presentation of complicated nonlinear systems could be present as equation (17) with use of fuzzy rules and simple linear dynamic models.

$$
\begin{aligned}
& R^{l}: \text { If } z_{1} \text { is } F_{1}^{l}, z_{2} \text { is } F_{2}^{l}, \ldots, z_{v} \text { is } F_{v}^{l} \\
& \text { Then } \\
& \qquad \begin{array}{l}
\frac{d}{d t} x(t)=\boldsymbol{A}_{l} x(t)+\boldsymbol{B}_{l} u(t) \\
y(t)
\end{array} \\
& \qquad \boldsymbol{C}_{l} x(t)+\boldsymbol{D}_{l} u(t) \quad l \in L:\{1,2, \ldots, m\}
\end{aligned}
$$

where $R^{l}$ is $l$-th fuzzy rule, $m$ is number of rules, $F_{j}^{l}$ is fuzzy sets, $x(t) \in \mathfrak{R}^{n}$ is a state variable vector of system, $u(t) \in \mathfrak{R}^{p}$ are system inputs and $y(t) \in \mathfrak{R}^{q}$ are outputs of linear system. Specification of linear dynamic model $l$ is determined with $\left(A_{l}, B_{l}, C_{l}, D_{l}\right)$ matrices and $z(t)=\left[z_{1}(t), z_{2}(t), \ldots, z_{v}(t)\right]$ are measurable variables of the system.

With use of fuzzy systems relations and use of singleton fuzzifier and product fuzzy inference and use of center average defuzzifier, Takagi-Sugeno fuzzy models will write as below.

$$
\begin{aligned}
& \frac{d}{d t} x(t)=\boldsymbol{A}(\mu) x(t)+\boldsymbol{B}(\mu) u(t) \\
& y(t)=\boldsymbol{C}(\mu) x(t)+\boldsymbol{D}(\mu) u(t)
\end{aligned}
$$

Where in equation (18)

$$
\begin{array}{lrrr}
\boldsymbol{A}(\mu)=\sum_{l=1}^{m} \mu_{l} \boldsymbol{A}_{l} & \boldsymbol{B}(\mu)=\sum_{l=1}^{m} \mu_{l} \boldsymbol{B}_{l} \quad \boldsymbol{C}(\mu)=\sum_{l=1}^{m} \mu_{l} \boldsymbol{C}_{l} \quad \boldsymbol{D}(\mu)=\sum_{l=1}^{m} \mu_{l} \boldsymbol{D}_{l} \\
\mu_{l}=\frac{\varepsilon_{l}(z)}{\sum_{i=1}^{m} \varepsilon_{i}(z)} & \varepsilon_{l}(z)=\prod_{i=1}^{v} F_{i}^{l}\left(z_{i}\right)
\end{array}
$$

Totally in this paper with use of obtained membership functions from modeling of PMSM, FOPID controller parameters will apply to the system.

\subsection{FUZZY NEURAL SYSTEMS}

As can be seen in the researcher reports, fuzzy logic has shown a high capability in control of complicated systems $[13,14]$. But in nonlinear system identification without expert supervisor will not have a high capability. On the contrary of this statement can be seen in neural networks $[15,16]$. For use of capabilities of these two approaches, fuzzy neural systems are formed [1719]. Figure 2 shows one the first combined structure of these two domains.

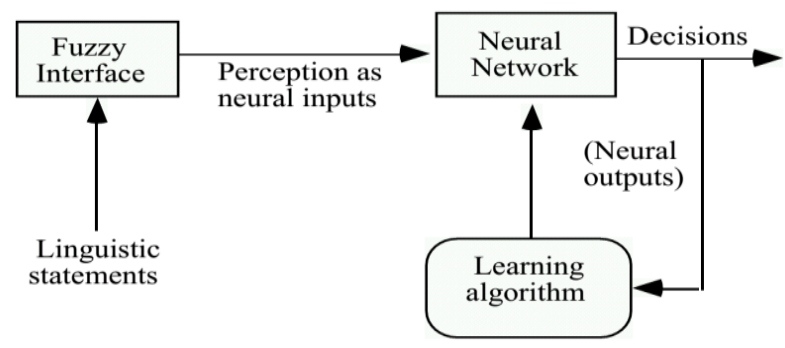

Figure 2. A fuzzy neural system 
As it is completely clear, it is possible to simply rewrite a neural network system as a neuro fuzzy system and conversely rewrite a Takagi-Sugeno fuzzy system as a fuzzy neural system with Gaussian neuron function (Figure 3).

According to figure 3, Takagi-Sugeno fuzzy system indicates a fuzzy system with four rules, two inputs and two membership function for each input which is shown as a neural system with five layers. Also, only first and forth layers are trainable. First layer includes membership parameters and forth layer is related to parameters that use in the fuzzy rules. It should be mentioned that if the parameters that used in forth layer is predefined, it is not necessary to train this part of neural network. Second layer neurons will use to calculate weight of membership function and third layer is assigned for normalization and last layer will use for final sum operation.
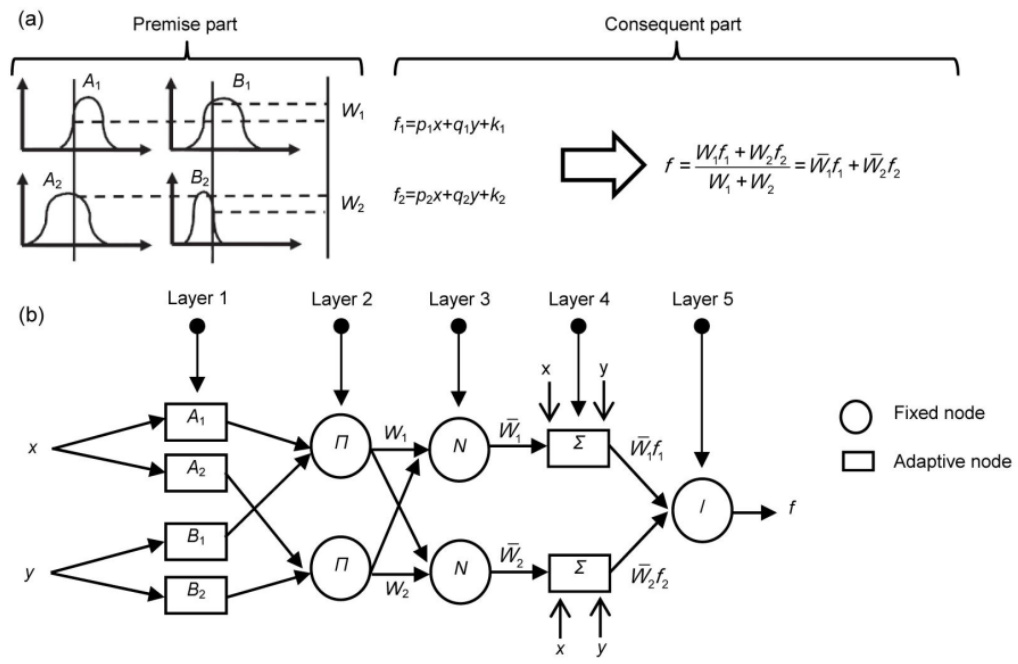

Figure 3. Modeling of Takagi-Sugeno fuzzy system as a neural network form

Usage of this proposed structure and training of neural network will add neural network capabilities to the fuzzy system which ANFIS is one of them [20]. In this paper, first TakagiSugeno fuzzy models for PMSM will propose that membership functions are Gaussian functions as a fuzzy neural form and it is possible to use advantages of neural network and fuzzy system. In neural network of proposed method, Levenberg marquardt is used that will explain in the next subsection.

\subsubsection{LeVenberg MarquardT Training Method}

This method is like as Newton method [21] and without need to calculate hessian matrix, uses the advantage of second order methods speed. If cost function is considered as a summation of error squares, hessian matrix will approximate as:

$$
H=J^{T} J
$$

and gradient will calculate as below form.

$$
g=J^{T} e
$$

where $J$ is a Jacobian matrix and includes first derivatives of error network. This algorithm will use a following procedure to estimate hessian matrix. 


$$
Q(k)=\left[J^{T} \cdot J+\mu I\right]^{-1}
$$

Therefore weight setting algorithm will be as bellowing form.

$$
W(k+1)=W(k)-\left(\left[J^{T} \cdot J+\mu I\right]^{-1}\right) \cdot\left(J^{T} \cdot e\right)
$$

When $J$ is zero, this method is equal to Newton method. When $J$ is selected large, this algorithm will equal to descent gradient method with very low step motion. Newton method is faster and more accurate and has a more coverage in error minimization; therefore the goal in this paper is possibly shifting toward of Newton method. Therefore $J$ after each successful step (reducing the cost function) will reduce and if each step increases the cost function, $J$ value will increase. As a result the cost function always will decrease

\section{EXPlaining The Structure Of Proposed Controller And SIMULATION RESULTS}

In this section first PMSM is modeled. This modeling is as a Takagi-Sugeno fuzzy system. Then for each of these fuzzy subsystems, a decentralized FOPID controller will design and finally general structure of controller will present. Results of proposed method in Simulink of MATLAB software show the capability of proposed controller mechanism.

\subsection{PMSM MODELLING}

$$
\begin{aligned}
v_{q s}^{r} & =r_{s} i_{q s}^{r}+\omega_{r} \lambda_{d s}^{r}+\frac{d}{d t} \lambda_{q s}^{r} \\
v_{d s}^{r} & =r_{s} i_{d s}^{r}-\omega_{r} \lambda_{q s}^{r}+\frac{d}{d t} \lambda_{d s}^{r} \\
\lambda_{q s}^{r} & =L_{q s} i_{q s}^{r} \\
\lambda_{d s}^{r} & =L_{d s} i_{d s}^{r}+\lambda_{m}^{r} \\
L_{q s} & =L_{l s}+L_{m q} \\
L_{d t} & =L_{l s}+L_{m d} \\
T_{e} & =\frac{3}{2} \frac{P}{2}\left[\lambda_{m}^{r} i_{q s}^{r}+\left(L_{d s}-L_{q s}\right) i_{q s} i_{d s}\right] \\
\frac{d}{d t} \omega_{r} & =\frac{1}{J}\left(T_{e}-B \omega_{r}-\frac{P}{2} T_{l}\right)
\end{aligned}
$$

where $B$ is a viscous friction coefficient and $J$ is a rotor equivalent inertia. With consideration surface mounted PMSM $L_{d s}=L_{q s}$ [22]. Non-linear equations of system could be presented as below.

$$
\begin{aligned}
& \frac{d}{d t} i_{q s}^{r}=\frac{1}{L_{q s}} v_{q s}^{r}-\frac{r_{s}}{L_{q s}} i_{q s}^{r}-\omega_{r} i_{d z}^{r}-\frac{\lambda_{m}^{r}}{L_{q s}} \omega_{r} \\
& \frac{d}{d t} i_{d s}^{r}=\frac{1}{L_{d s}} v_{d s}^{r}-\frac{r_{s}}{L_{d s}} i_{d s}^{r}+\omega_{r} i_{q s}^{r} \\
& \frac{d}{d t} \omega_{r}=\frac{3 P^{2} \lambda_{m}^{r}}{4 J} i_{q s}^{r}-\frac{B}{J} \omega_{r}-\frac{P}{2 J} T_{l}
\end{aligned}
$$

In this system, the inputs are $v_{q s}^{r}$ and $v_{d s}^{r}$ and outputs are $i_{q s}^{r}, i_{d s}^{r}$ and $\omega_{r}$. In the proposed method, for control mechanism, the purpose of control is torque $T_{e}$ and flux $\lambda_{d s}^{r}$ which can be calculated with consideration of simple estimator based on system variables [22]. 


$$
\begin{aligned}
& \lambda_{d s}^{r}=L_{d s} i_{d s}^{r}+\lambda_{m}^{r} \\
& T_{e}=\frac{3}{2} \frac{P}{2} \lambda_{m}^{r} i_{q s}^{r}
\end{aligned}
$$

In is clear that the system in equation (24) is nonlinear. This system can be presented with the Takagi-Sugeno fuzzy system with $m$ rules as equation (26) [23].

$$
\begin{aligned}
& \frac{d}{d t} \omega_{r}=\frac{3 P \lambda_{m}^{r}}{4 J} i_{q s}^{r}-\frac{B}{J} \omega_{r}-\frac{P}{2 J} T_{l} \\
& \frac{d}{d t} i_{q s}^{r}=\frac{1}{L_{q s}} v_{q s}^{r}-\frac{r_{s}}{L_{q s}} i_{q s}^{r}-\left(\sum_{i=1}^{m} h_{i}\left(\omega_{r}\right) W_{i}\right) i_{d s}^{r}-\frac{\lambda_{m}^{r}}{L_{q s}} \omega_{r} \\
& \frac{d}{d t} i_{d s}^{r}=\frac{1}{L_{d s}} v_{d s}^{r}-\frac{r_{s}}{L_{d s}} i_{d s}^{r}+\left(\sum_{i=1}^{m} h_{i}\left(\omega_{r}\right) W_{i}\right) i_{q s}^{r}
\end{aligned}
$$

For this fuzzy system, membership functions will calculate as below.

$$
h_{i}\left(\omega_{r}\right)=\frac{e^{-\mu_{i}\left(\omega_{r}-W_{i}\right)^{2}}}{\sum_{i=1}^{m} e^{-\mu_{i}\left(\omega_{r}-W_{i}\right)^{2}}}
$$

With consideration $\omega$ as a neural network input, $\sqrt{\mu_{i}}$ as a weighting functions, $W_{i}$ as a predefined biased values form and consideration Gaussian function as a neuron operational function, above system could present as a form of neuro fuzzy system and with use of neural network online training capabilities benefit, closed loop system efficiency will improve.

\subsection{CONTROL SYSTEM}

Nowadays in many complicated systems, design of proper controller and implementable is very important. With respect to capability of fractional order systems in identification of complicated systems as a form of first fractional order system with delay, usage of FOPID had a proper results. Also in the recent years, fuzzy neural controllers are widely considered. The reason of this usage is success of fuzzy neural controller in the uncertain and complicated systems. Usage of fuzzy neural supervisory system in FOPID controller is the main idea in this paper and improves the operation of control system for PMSM. General block diagram of proposed method is shown in figure 4.

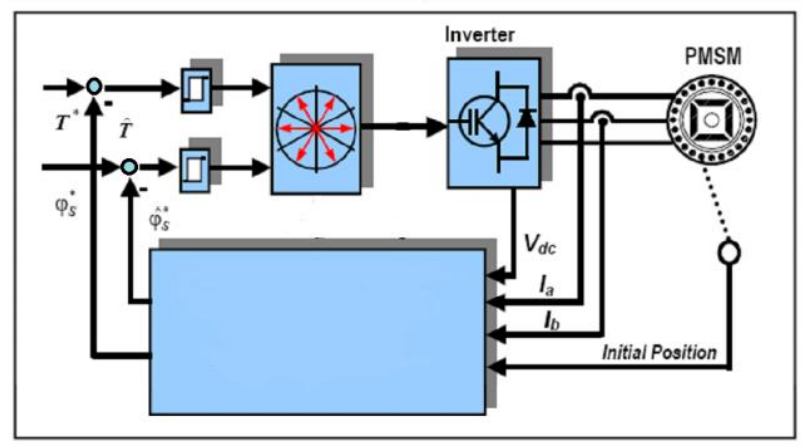

Figure 4. General proposed block diagram of direct current control of PMSM 


\subsubsection{CONTROL BLOCK DIAGRAM}

In this paper, proposed control block diagram of figure 5 for torque direct control of PMSM is used. In this structure according to Takagi-Sugeno fuzzy system model, coefficient of FOPID with use of GA will calculate as an offline form. After determination of FOPID coefficients, minimization of error absolute integral will carry out with GA. Then in the online condition, coefficients of FOPID will correct with weighting functions in neuro fuzzy system and combine together and finally proper control signal will apply to the system. As it is clear, Figure 5 is a control part of block diagram presented in Figure 4. Proposed control block diagram is explained at continuation of paper.

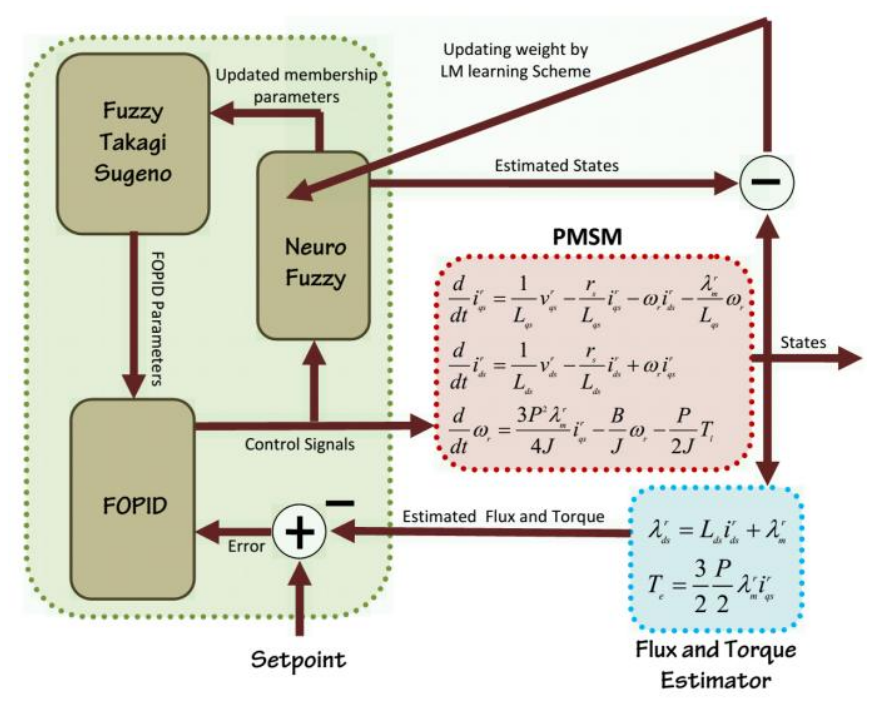

Figure 5. Proposed control structure

\subsubsection{Fuzzy neural block diagram}

This block will calculate membership function parameter. In fact this block includes modeling of PMSM. The equations of PMSM as a Takagi-Sugeno fuzzy system is as:

$$
\begin{aligned}
& R^{l}: \text { If } \omega_{r} \text { is } W_{i} \text { Then } \quad \frac{d}{d t} \omega_{r}=\frac{3 P \lambda_{m}^{r}}{4 J} i_{q s}^{r}-\frac{B}{J} \omega_{r}-\frac{P}{2 J} T_{l} \\
& \frac{d}{d t} i_{q s}^{r}=\frac{1}{L_{q s}} v_{q s}^{r}-\frac{r_{s}}{L_{q s}} i_{q s}^{r}-\left(W_{i} \times i_{c t}^{r}\right)-\frac{\lambda_{m}^{r}}{L_{q s}} \omega_{r} \\
& \frac{d}{d t} i_{d k}^{r}=\frac{1}{L_{c k}} v_{c k}^{r}-\frac{r_{s}}{L_{d k}} i_{d k}^{r}+\left(W_{i} \times i_{q s}^{r}\right) \quad i \in L:\{1,2, \ldots, 8\}
\end{aligned}
$$

where $W_{i}$ has a predefined which PMSM system in linearized around it. For this fuzzy system, membership function is as equation (27). In this paper values are selected as a linear form between $0 \leq \omega_{r} \leq 400$ system working points. It would be mentioned that usage of more working region, although will increase modeling precision but will increase complexity. Therefore balance between complexity and precision should be considered which with try and error the eight regions are considered. Other problem is correct selection of membership function parameters $\mu_{i}$ to minimize error between differentiations of main system and modeled state variables. For proper selection of $\mu_{i}$, as explained in section 4.2, fuzzy model is presented with neural network with $\omega_{r}$ input and eight neurons in the first to fourth layers and one neuron in the fifth layer. 
According to figure 6 , in this neural network, there is not weight parameter and only $\mu$ parameter in the first layer is adjustable. In figure $6, F_{i}$ is an estimation of PMSM state variables differentiation vector which will calculate with Takago-Sugeno fuzzy system (equation (28)). According to figure 6, output of Fuzzy neural block diagram is membership function parameters to use for Takagi-Sugeno fuzzy system (Figure 5).

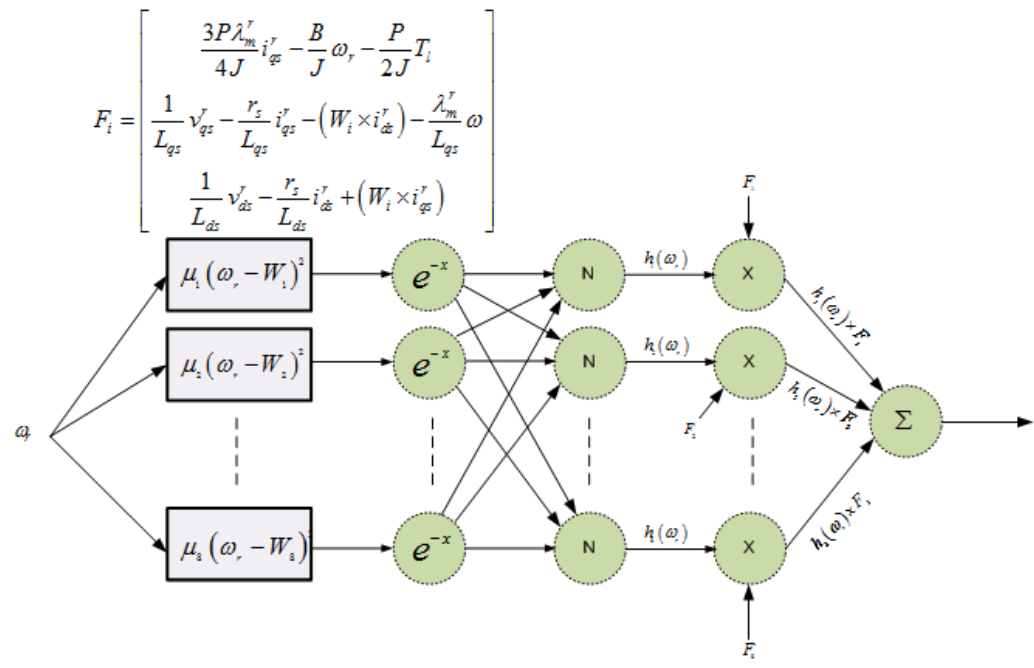

Figure 6. PMSM modeling as a fuzzy neural network form

\subsubsection{Fuzzy block diagram}

This block diagram based on membership function parameters which are calculated from neuro fuzzy and coefficients that are obtained for each linear region of GA, will calculate FOPID parameters. The rules of fuzzy block diagram are as below.

$$
R^{l} \text { :If } \omega_{r} \text { is } W_{i} \text { Then } K_{P}=K_{P_{W_{i}}} K_{I_{\alpha_{j}}}=K_{I_{\alpha_{j} w_{i}}} K_{D_{\beta_{j}}}=K_{D_{\beta_{j} w_{i}}} i \in L:\{1,2, \ldots, 8\} \quad j \in L
$$

$K_{P_{W_{i}}}, K_{I_{\alpha_{j} W_{i}}}$ and $K_{D_{\beta_{j} W_{i}}}$ are parameters which are calculated for eight regions with GA.

\subsubsection{Genetic Algorithm (GA)}

In simulation, initial population is considered 200 genes and with respect to error square integral, membership functions will optimize in the eight regions that is considered in our simulation.

\subsubsection{Implemented block diagram in MATLAB software}

Figure 7 shows the proposed implemented control structure. As it is clear, this structure consists of 6 general parts.

Parts 1 is used for PMSM modeling.

Part 2 is used to apply membership function values to the fractional controller.

Part 3 will calculate error function according to the defined criteria.

Part 4 includes calculation of fractional differentiation and integral.

Part 5 shows the degree of membership functions and its output is control signals.

Torque and flux will estimate in the part 6. 


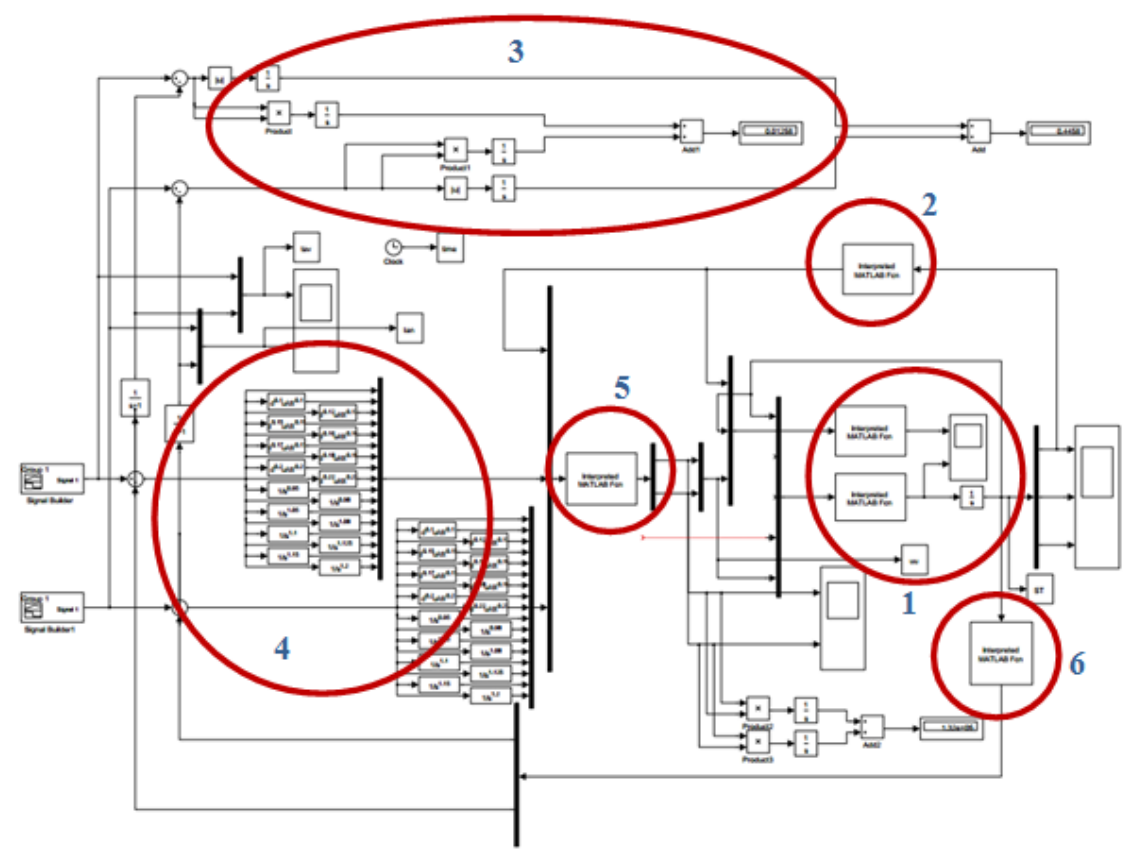

Figure 7. Proposed control diagram in MATLAB Simulink software

\subsubsection{Steps of proposed algorithm}

Generally the proposed algorithm includes following steps.

Step 1: Design of FOPID controller coefficients with use of GA for all the Takagi-Sugeno fuzzy subsystems and motor identification in different working points.

Step 2: Selection of initial state for fuzzy neural system to create the membership functions.

Step 3: Applying control signal to the system and changing system state variables.

Step 4: Setting fuzzy neural network parameters according to error between real system and simulated system based on Levenberg marquardt training algorithm.

Step 5: Calculation of torque and flux in the estimator block.

Step 6: Calculation of error, FOPID controller parameters and control signal.

Step 7 : Go back to the step 3 and continue till end of the simulation.

\section{SimUlation}

In this section results of simulation for proposed controller will present to evaluate capabilities of proposed method Fuzzy Neural FOPID named FNFOPID. PMSM parameters used in the simulation are as below [24].

$$
\begin{aligned}
& \omega_{\text {rated }}=1850(R P M), \quad T_{\text {rated }}=3.87(\mathrm{~N} . M), \quad P=12, R_{s}=0.99(\Omega), L_{s}=5.82(\mathrm{mH}), \\
& \lambda_{m}=7.915 \times 10^{-2}(\mathrm{~V} . \mathrm{s} / \mathrm{rad}), J=12.075 \times 10^{-4}\left(\mathrm{Kg} \cdot \mathrm{m}^{2}\right), B=3 \times 10^{-4}(\mathrm{~N} . \mathrm{m} . \mathrm{s} / \mathrm{rad})
\end{aligned}
$$

Eight fuzzy membership functions are selected in cluster center 50, 100, 150, 200, 250, 300, 350 and 400 radians per second. Then FOPID will calculate for each condition of system. To evaluate FNFOPID method, this method is tested in three conditions nominal, disturbance elimination and noise and uncertainly. Figures 8 to 11 show function of proposed mechanism FNFOPID in tracking desired signal which evaluate completely perfect. 
International Journal Of Instrumentation And Control Systems (IJICS) Vol.7, No.2, April 2017

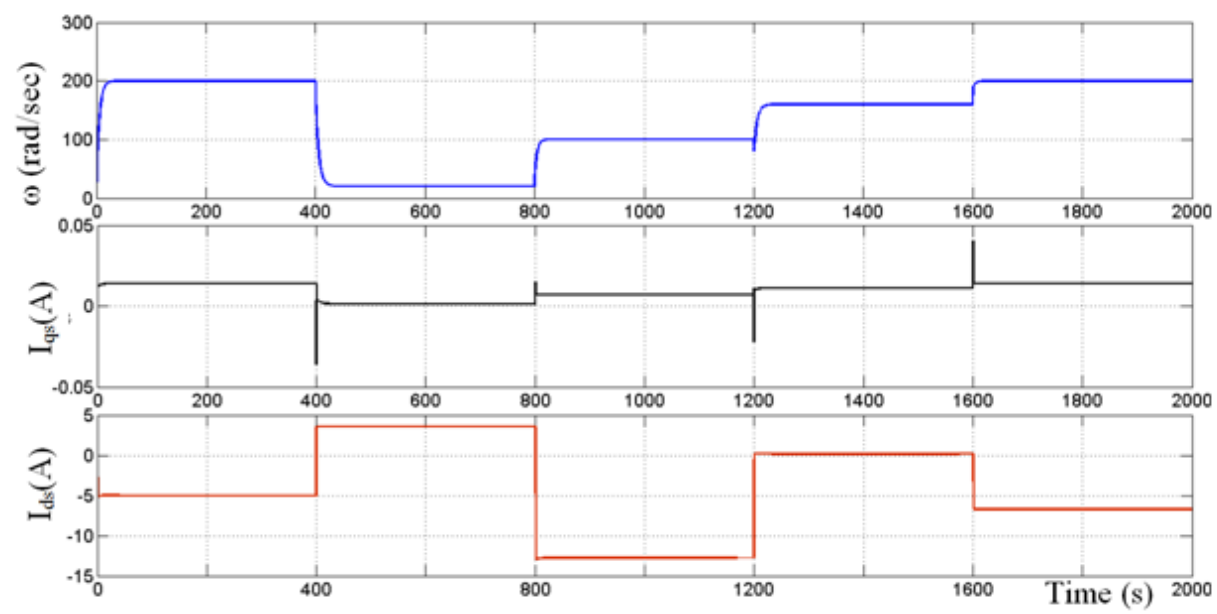

Figure 8. FNFOPID state variables variations in tracking reference signal

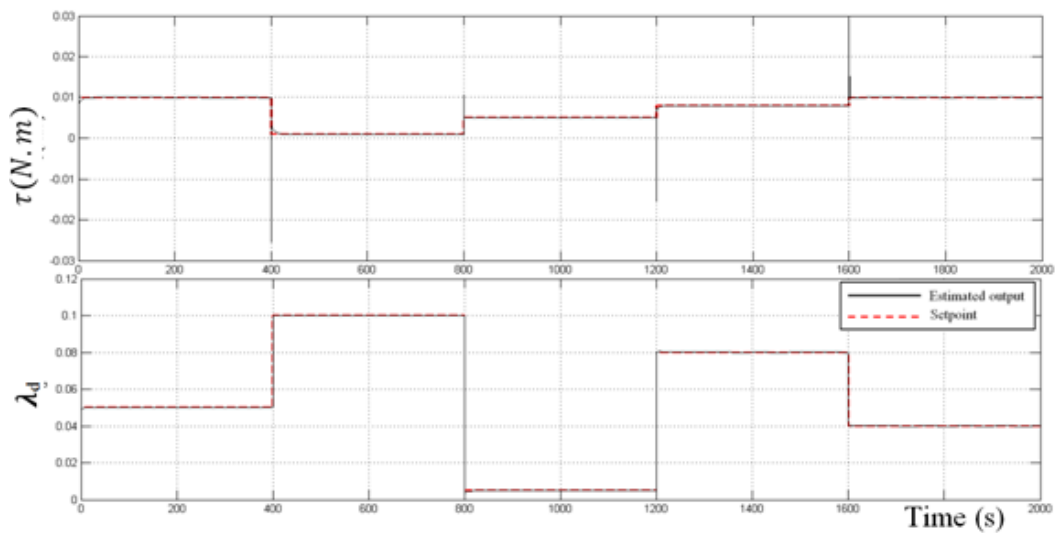

Figure 9. FNFOPID outputs variations and desired values
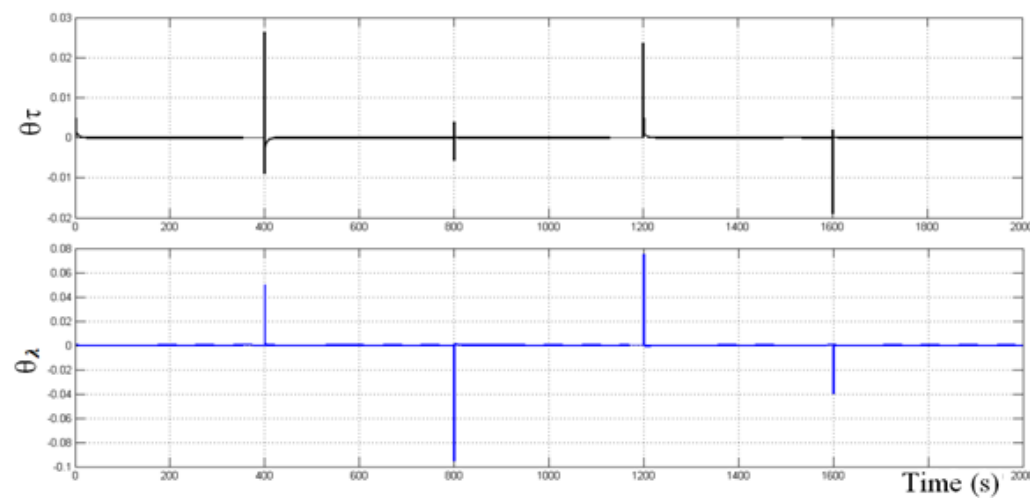

Figure 10. Error value between FNFOPID outputs and desired values 
International Journal Of Instrumentation And Control Systems (IJICS) Vol.7, No.2, April 2017

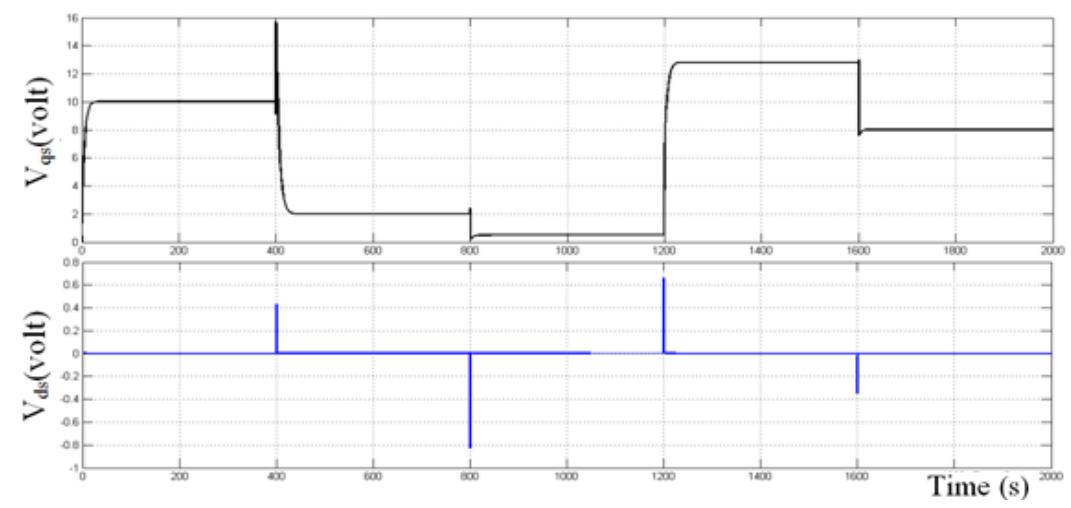

Figure 11. Curve of FNFOPID control signal variations

Figure 12 shows function of FNFOPID in presence of step disturbance. Disturbance is a pulse signal with period of 400 second and amplitude of 8 and added to second part of equation (28). Results show the capability of FNFOPID in elimination of disturbance effect.

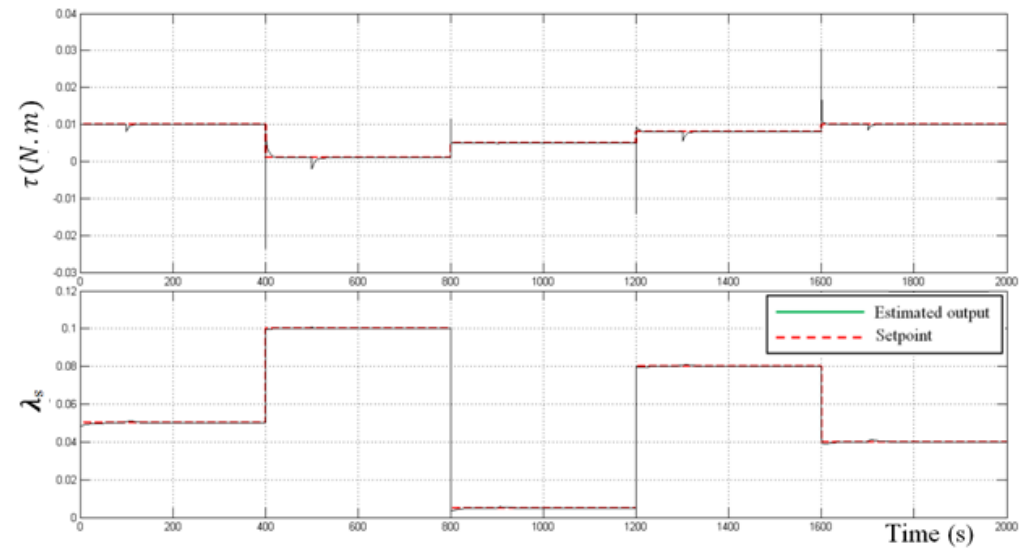

Figure 12. FNFOPID outputs variations and desired values in presence of step disturbance

Figure 13 shows function of FNFOPID in presence of noise and uncertainty. Results show the capability of FNFOPID in existence of noise and uncertainty.

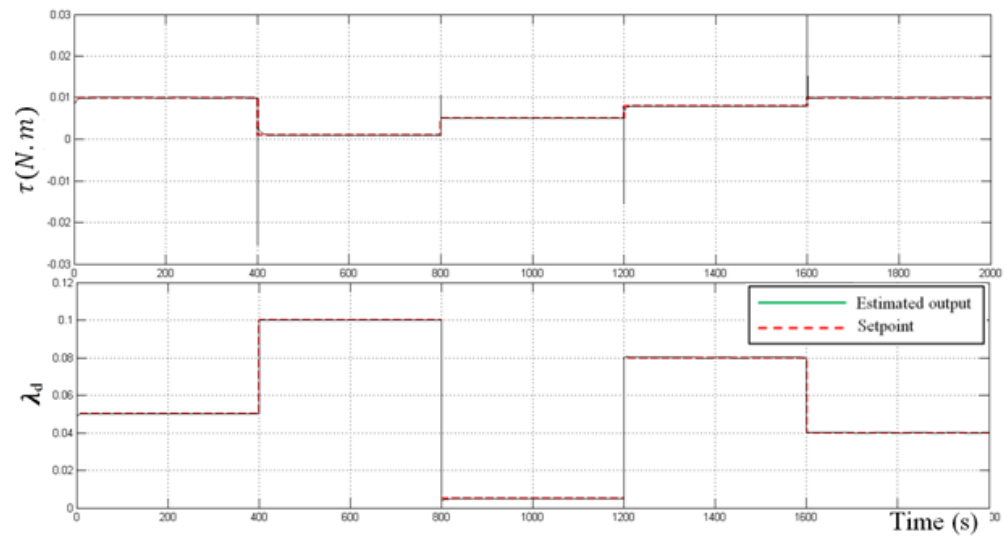

Figure 13. FNFOPID outputs variations and desired values in presence of noise and uncertainty 
International Journal Of Instrumentation And Control Systems (IJICS) Vol.7, No.2, April 2017

Figures 14 to 16 compare FNFOPID method with FOPID. Although from these figures precise comparison is not possible but figure 14 shows a closed loop system tacking that shows FNFOPID is better than FOPID.

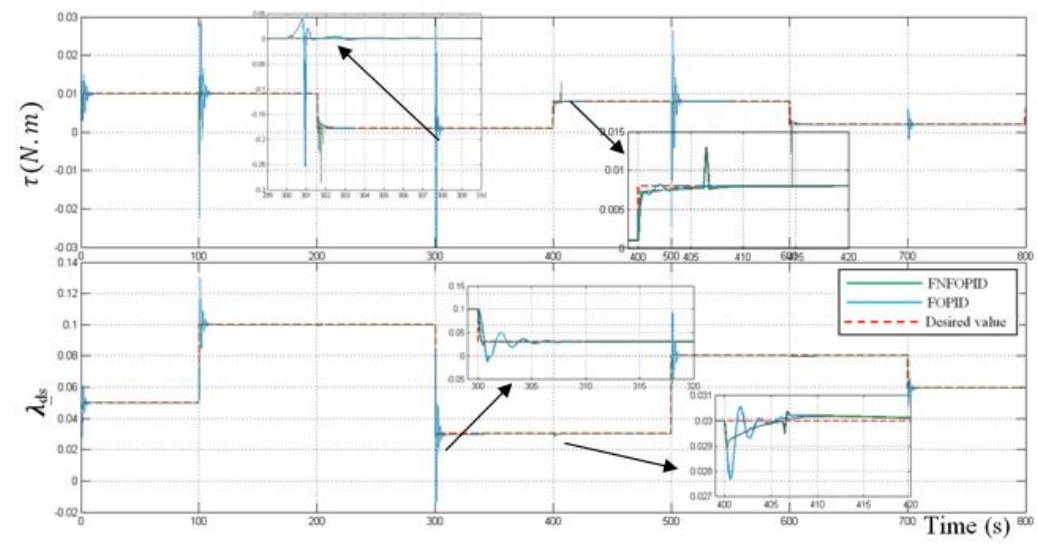

Figure 14. Comparison tracking of FNFOPID and FOPID outputs

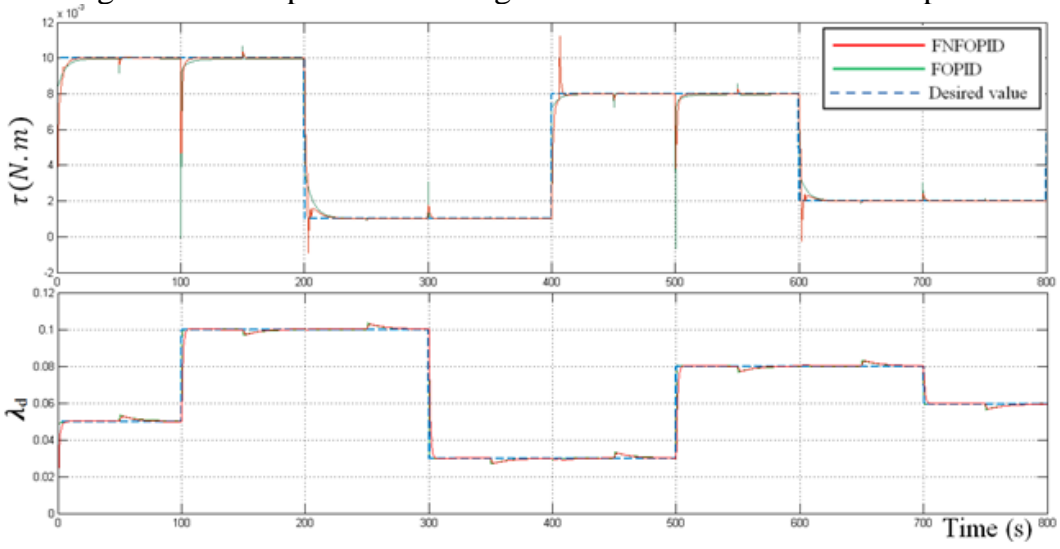

Figure 15. Comparison of tracking of FNFOPID and FOPID in presence of disturbance

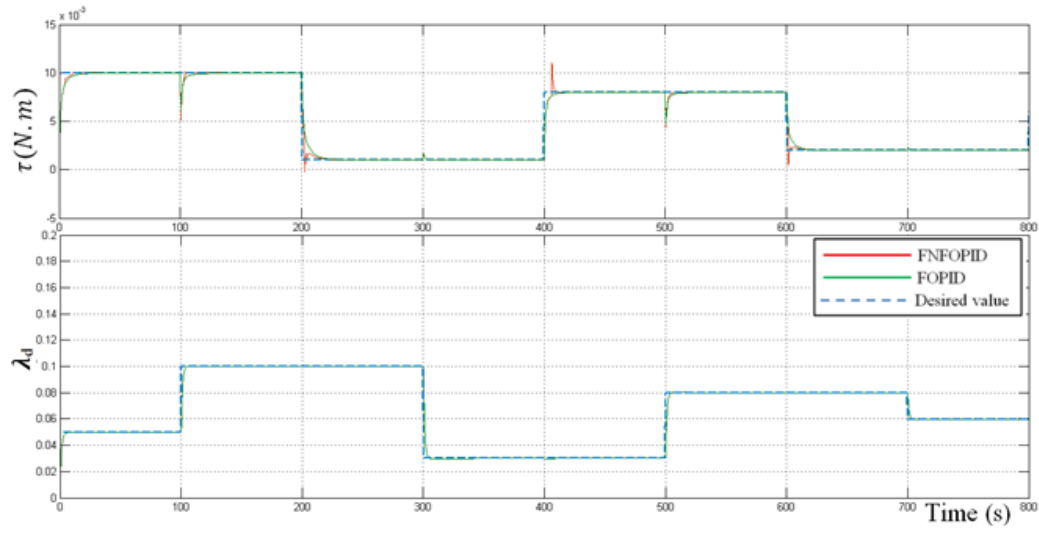

Figure 16. Comparison of tracking of FNFOPID and FOPPID in uncertainty and noise condition

Table 1 shows the comparison of proposed method results with FOPID results in two criteria that are Integral Square Error (ISE) and Integral Absolute Error (IAE). 
These comparisons are carried out in three states; nominal state, in existence of disturbance and in occurrence of noise and uncertainty. With respect to the obtained results, it is completely clear that the proposed control method in this paper in all considered conditions in more efficient than FOPID controller and has a less error.

According to table 1 and IAE criterion, proposed method in the worse condition that is in the nominal case $5.27 \%$ and in the best condition in presence of noise and uncertainty $11.54 \%$ error will decrease. With respect to ISE criterion, proposed method in the worse condition in presence of disturbance has $8.66 \%$ error reduction and in the best condition in the nominal case has $15.25 \%$ improvement in control of system.

Table 1. Numerical comparison of proposed technique with FOPID controller

\begin{tabular}{|c|c|c|c|c|}
\hline \multirow{3}{*}{ Method } & \multicolumn{2}{|c|}{ Criteria } & \multicolumn{2}{|c|}{ Percentage of improvement (\%) } \\
\hline & IAE & ISE & IAE & ISE \\
\hline & $\int|e| d t$ & $\int e^{2} d t$ & $\int e \mid d t$ & $\int e^{2} d t$ \\
\hline FNFOPID (Nominal Form) & 0.41992 & 0.01256 & 5.26767 & 15.24970 \\
\hline FNFOPID with step disturbance & 0.52383 & 0.01719 & 6.27650 & 8.66100 \\
\hline FNFOPD with noise and uncertainty & 0.43235 & 0.01431 & 11.5378 & 11.0628 \\
\hline FOPID (Nominal Form) & 0.44327 & 0.01482 & & \\
\hline FOPID with step disturbance & 0.55891 & 0.01882 & & \\
\hline FOPD with noise and uncertainty & 0.48874 & 0.01609 & & \\
\hline \multicolumn{3}{|c|}{ Average of improvement } & 7.694000 & 11.657800 \\
\hline
\end{tabular}

\section{Conclusion}

In this paper, new mechanism for torque direct control of PMSM is proposed. Simulations showed the capability of proposed mechanism. This proposed method in extendable for many nonlinear systems. Usage of fuzzy neural system capability is completely evident that with proper combination of predesigned coefficients has a good performance in tracking, elimination of disturbance and robustness against system parameter variations. In this paper, operation of proposed control method to control PMSM is compared with FOPID in the nominal, presence of disturbance and noise and uncertainty conditions. Results show the capability of proposed mechanism.

\section{REFERENCES}

[1] A. B. Dehkordi, A. M. Gole \& T. L. Maguire, (2005), "Permanent Magnet Synchronous Machine Model for Real- Time Simulation”, International Conference on Power Systems Transients (IPST), Montreal, Canada.

[2] Perera, P.C., Blaabjerg, F., Pedersen, J.K. \& Thogersen, P., (2003), "A sensorless, stable V/f control method for permanent-magnet synchronous motor drives", IEEE Transactions on Industry Applications, Vol. 39, No. 3, pp 783-791.

[3] Mohamed, Y.A.R.I., (2007), "Design and implementation of a robust current-control scheme for a PMSM vector drive with a simple adaptive disturbance observer", IEEE Transactions on industrial electronics, Vol. 54, No. 4, pp 1981-1988.

[4] Ting-ting Liu, Yu Tan, Gang Wu \& Shu-mao Wang, (2009), "Simulation of PMSM vector control system based on Matlab/Simulink", International Conference on measuring technology and Mechatronics Automation, Vol. 2.

[5] Wang, C.M, Y.J. Ji, H. Luan \& Z.E. Zhang, (2009), "Simulation of PMSM vector control system based on Matlab/Simulink", J. Jilin University, Vol. 27, pp 17-22. 
[6] Hongping, J. \& Yikang, H., (2006), "Variable structure sliding mode control for PMSM DTC", Transactions of China Electrotechnical Society, Vol. 21, No. 1, pp 1-6.

[7] Sun, D. \& Yikang HE, (2005), "A modified direct torque control for PMSM under inverter fault", International Conference on Electrical Machines and Systems, pp 2473.

[8] Jalali A.A. \& S. Khosravi, (2011), "Tuning of FOPID Controller Using Taylor Series Expansion", International Journal of Scientific \& Engineering Research, Vol. 2, No. 5.

[9] Yeroglu C., \& N. Tan, (2011), "Note on fractional-order proportional-integral-differential controller design", IET Control Theory \& Applications, pp 1978-1989.

[10] Monje, C.A., Chen, Y.Q., Vinagre, B.M., Xue, D., \& Feliu, V., (2010), "Fractional-order Systems and Controls: Fundamentals and Applications", Springer, London. ISBN: 978-1-84996-334-3.

[11] Wang Lin \& Zhong Chongquan, (2015), "Design of optimal fractional-order PID controllers using particle swarm optimization algorithm for DC motor system", Advanced Information Technology, Electonic and Automation Control Conference (IAEAC), Chongqing, China.

[12] Yen, J., Wang, L. \& C. Wayne Gillespie, (1998), "Improving the interpretability of TSK fuzzy models by combining global learning and local learning", IEEE Transaction on Fuzzy systems, No. 4, pp 530-537.

[13] Sun, D., Yikang HE \& Jian Guo ZHU, (2004), "Fuzzy Logic Direct Torque Control for Permanent Magnet Synchronous Motors", Proceedings of the 5th World Congress on Intelligent Control and Automation, Hangzhou. P.R. China.

[14] Sun Dan., He Yikang \& Zhi Dawei, (2003), "Direct Torque Control of a Permanent Magnet Synchronous Motor Based on Fuzzy Logic", Transactions of China Electrotechnical Society.

[15] Rahman, M.A. \& M.A.Hoque, (1998), "On-line adaptive artificial neural network based vector control of permanent magnet synchronous motors", IEEE Transactions on Energy Conversion, Vol. 13, No. 4, pp 311-318.

[16] Sun, D. \& J. Meng, (2006), "A Single Neuron PID Controller Based PMSM DTC Drive System Fed by Fault Tolerant 4-Switch 3-Phase Inverter", IEEE international conference on industrial electronics and applications.

[17] Nauck, D., Klawon, F. \& R. Kruse, (1997), Foundations of Neuro-Fuzzy Systems, J. Wiley \& Sons.

[18] Czogala, E. \& J. Leski, (2000), Neuro-Fuzzy Intelligent Systems, Studies in Fuzziness and Soft Computing, Springer Verlag, Germany.

[19] Figueiredo, M. \& F. Gomide, (1999), "Design of Fuzzy Systems Using Neuro-Fuzzy Networks", IEEE Transactions on Neural Networks, No. 4, pp 815-827.

[20] LinT. C., \& C. S. Lee., (1991), "Neural Network Based Fuzzy Logic Control and Decision System", IEEE Transactions on Computers, No. 12, pp 1320-1336.

[21] Ranganathan A., (2004), The Levenberg-Marquardt Algorithm, Honda Research Institute, USA. 8 June.

[22] Munoz, D. V., (2011), Design, Simulation and Implementation of a PMSM Drive System, Ph.D. thesis, Chalmers University of Technology, Goetenberg, Sweden.

[23] Schnitman, L., Felippe de Souza, J. A. M. \& T. Yoneyama, (2001), "Takagi-Sugeno-Kang fuzzy structures in dynamic system modeling", IASTED International Conference on Control and Application, Vancouver, pp 34-38.

[24] Vu, N. T. T., Choi, H. H., Kim, R. Y \& Jung, J. W., (2012), "Robust speed control method for permanent magnet synchronous motor", IET Electronic Power Applications, pp 399-411.

\section{AUTHORS}

Gholamreza Farahani received his BSc degree in electrical engineering from Sharif University of Technology, Tehran, Iran, in 1998 and MSc and PhD degrees in electrical engineering from Amirkabir University of Technology (Polytechnic), Tehran, Iran in 2000 and 2006 respectively. Currently, he is an assistant professor in the Institute of Electrical and Information Technology, Iranian Research Organization for Science and Technology (IROST), Iran. His research interest is control systems especially neural networks and fuzzy systems.

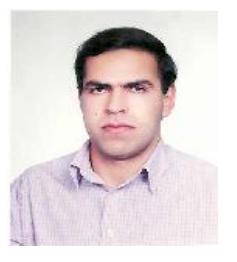

